\title{
Complicações Hiperglicêmicas Agudas no Diabetes Melito Tipo 1 do Jovem
}

\begin{abstract}
RESUMO
A principal complicação hiperglicêmica no diabetes melito tipo 1 (DM1) é a cetoacidose diabética (CAD). Embora variações nos protocolos possam ocorrer, os princípios básicos que norteiam o tratamento devem ser os mesmos. A recuperação inicial da capacidade circulatória, com a infusão rápida de solução salina na dose de $20 \mathrm{~mL} / \mathrm{kg}$, que pode ser repetida, é o ponto de partida para o tratamento. A partir daí, a reposição de volume é relativamente lenta, e o objetivo principal é corrigir gradualmente os distúrbios metabólicos instalados, sem ocasionar variações muito intensas e muito rápidas na osmolalidade, fator de risco para complicações. Atenção ao desenvolvimento de edema cerebral que, uma vez suspeitado, deve ser imediatamente corrigido, sob pena de óbito ou seqüelas neurológicas. A administração de insulina ultra-rápida, por via subcutânea, mostra-se eficaz e simplifica o atendimento do paciente. A CAD é uma situação grave, ainda com alta mortalidade, e seu tratamento deve ser dirigido aos pontos principais que levaram ao quadro clínico, com correções graduais, sob risco de se agravar o quadro. (Arq Bras Endocrinol Metab 2008;52/2:367-374)
\end{abstract}

Descritores: Cetoacidose diabética; Estado hiperglicêmico hiperosmolar; Edema cerebral; Acidose metabólica; Desidratação

\section{ABSTRACT}

\section{Hyperglycemic Acute Crisis in Type 1 Diabetes Mellitus in Youth}

Diabetic ketoacidosis (DKA) is the main hyperglycemic complication in type 1 Diabetes Mellitus (DM1). The basic principles in treatment have to be followed carefully. The patient with DKA has a very deep volume depletion. To restore the circulatory capacity is the first step. From this point on, the restoration of the lost fluids is slow, around $1 \%$ per hour, aiming at the correction of the metabolic disturbance already on and avoiding great fluctuations in osmolality, which increases the risk of having complications. Attention to the development of cerebral edema, which, once suspected, deserves an urgent treatment plan, trying to avoid neurologic sequelae or even death. Subcutaneous ultra-rapid insulin has been demonstrated to be efficient and easier to use. As the perfusion gets improved and the levels of insulin increase, the lipolysis is blocked, as well as the generation of ketones and so the acidemia tends to be solved. DKA is still a high-mortality condition. And to be in a hurry frequently leads to neurologic sequelae and even to a fatal outcome. (Arq Bras Endocrinol Metab 2008;52/2:367-374)

Keywords: Diabetic ketoacidosis; Hyperglycemic hyperosmolar state; Cerebral edema; Metabolic acidosis; Dehydration revisõo

\author{
Durval Damiani \\ DaNIEL DamianI
}

Unidade de Endocrinologia

Pediátrica do Instituto da

Criança do Hospital das Clínicas da Faculdade de Medicina da

Universidade de São Paulo (FMUSP) (DD); Curso de Biomedicina da Universidade Nove de Julho (DD), São Paulo, SP, Brasil.

Recebido em 03/12/2007 Aceito em 12/12/2007 


\section{INTRODUÇÃO}

A PRINCIPAL CAUSA DE MORTALIDADE no diabetes melito tipo l (DMl) é a cetoacidose diabética (CAD), mais especialmente quando ocorre o temível edema cerebral. Em 25\% dos casos, é a CAD a primeira manifestação do DM e o não reconhecimento desta condição promove progressiva deterioração metabólica que pode levar a sérias seqüelas ou mesmo ao óbito. Apesar de ser uma complicação inicial relativamente freqüente no DMl, ela não é exclusiva dessa forma de DM, pois pacientes portadores de diabetes melito tipo 2 (DM2) também podem abrir seu quadro com CAD. Já os estados hiperosmolares hiperglicêmicos (HHS) ocorrem quase exclusivamente com o DM2 (1). Não se pode esquecer que, diante do aumento importante da obesidade, casos de DM2 têm se tornado mais freqüentes em faixas etárias, em que, há algum tempo, essa forma de DM não ocorria. Assim, o aparecimento de casos de HHS pode começar a ocorrer e deve-se ficar atentos a esta possibilidade, para se tomar condutas adequadas a cada caso.

Entre 190 pacientes com DM2 estudados por Fourther e cols., oito apresentaram-se em HHS (4,2\%) (2). O HHS caracteriza-se por concentrações glicêmicas $>600$ $\mathrm{mg} / \mathrm{dL}$, bicarbonato $>15 \mathrm{mmol} / \mathrm{L}$, ausência ou traços de cetonúria e cetonemia, osmolalidade sérica $>320$ $\mathrm{mOsm} / \mathrm{kg}$, estupor ou coma (3).

No DMl, a descompensação em CAD pode ser a primeira manifestação da doença, uma grave condição clínica que deve ser tratada em unidade de terapia intensiva e por equipe habituada a tratar esse tipo de complicação diabética. Ainda hoje, $5 \%$ a $10 \%$ dos casos de CAD vão a óbito. Portanto, de um correto conhecimento fisiopatológico desta condição depende a tomada de decisões de modo correto e a possibilidade de se lidar com esses casos com o menor risco possível para o paciente.

Quando um paciente com DMl chega a um estado de $\mathrm{CAD}$, uma série de alterações metabólicas já ocorreram e vários mecanismos de defesa já foram postos em jogo para tentar manter o "meio interno" e evitar um desfecho fatal. Portanto, ao tratar o paciente em CAD não se deve esquecer que o respeito ao "desmonte" desses processos que se instalaram para fazer frente ao desarranjo metabólico inicial é fundamental para um bom desfecho do caso. Para, por exemplo, normalizar uma glicemia em torno de $800 \mathrm{mg} / \mathrm{dL}$ em uma ou duas horas, se estará provocando um deslocamento de fluidos de um compartimento para outro de maneira tão intensa que o edema cerebral certamente ocorrerá, com todas as suas conseqüências e com alta mortalidade (4).
Há várias definições de $\mathrm{CAD}$, modificando-se a concentração glicêmica de $300 \mathrm{mg} / \mathrm{dL}$ para $200 \mathrm{mg} /$ $\mathrm{dL}$ e mesmo aceitando-se a presença de CAD sem hiperglicemia, em casos parcialmente tratados ou em crianças em jejum prolongado. Apesar de se reconhecer que pode haver CAD sem hiperglicemia, ou mesmo sem acidose (em casos de vômitos intensos, uso de diuréticos, síndrome de Cushing ou hiperaldosteronismo) ou mesmo sem cetose, a definição clássica, que se utilizou em nosso estudo é: $\mathrm{pH}<7,3$ ou concentração de bicarbonato sérico $<15 \mathrm{mmol} / \mathrm{L}$, concentração de glicose $>300 \mathrm{mg} / \mathrm{dL}$, cetonemia superior a $3 \mathrm{mmol} / \mathrm{L}$ (referente a nível de beta-hidróxibutirato - $\beta$-HM $\beta$ ) ou presença de cetonúria (esta, disponível em qualquer serviço de emergência) (5). Quando se utiliza a avaliação urinária de cetonas, com tiras reagentes, ressaltar que é o acetoacetato que é detectado e que a proporção de $\beta$-HM $\beta$ para acetoacetato é de 8 a 10:1. O que quer dizer que, mesmo após ter cessado a produção de corpos cetônicos, por algum tempo a cetonúria estará presente, em virtude da conversão de $\beta$-HM $\beta$ a acetoacetato e isso não significa que o quadro de $\mathrm{CAD}$ não tenha melhorado ou exija novas abordagens terapêuticas, um erro freqüentemente cometido. Atualmente, dispõe-se de tiras reagentes para avaliação sangüínea de $\beta$-HM $\beta$, que podem servir de monitorização da evolução metabólica do paciente em CAD.

\section{O PROCESSO FISIOPATOLÓGICO DOS ESTADOS HIPERGLICÊMICOS}

Tanto a CAD quanto os HHS ocorrem quando há déficit de insulina, absoluto ou relativo, estimulando a liberação de hormônios contra-reguladores, como glucagon, catecolaminas, cortisol e hormônio de crescimento. Essas respostas hormonais estimulam a produção hepática e renal de glicose enquanto reduzem sua utilização nos músculos, no fígado e no tecido adiposo. Como conseqüência, ocorre hiperglicemia, liberação de ácidos graxos a partir do tecido adiposo (lipólise) e oxidação hepática de ácidos graxos a corpos cetônicos. Nos estados de HHS, apesar de a insulina ser incapaz de evitar a hiperglicemia, por causa de um estado de resistência à insulina, é suficiente para bloquear a lipólise e a cetogênese.

A hidrólise de triglicérides no adipócito libera ácidos graxos de cadeia longa e glicerol para a circulação. Dois terços desses ácidos graxos mobilizados são utilizados diretamente e o terço restante é captado pelo fígado para conversão a corpos cetônicos. Em condições 
anabólicas normais, o fígado produz pequeníssimas quantidades de corpos cetônicos (ácido acetoacético e $\beta-\mathrm{HM} \beta)$. Os ácidos graxos dietéticos entram no hepatócito, são esterificados a triglicérides e transportados na circulação como VLDL (very low density lipoproteins). Para que se desenvolva uma cetose importante, é necessário o aporte de ácidos graxos livres (FFA) ao fígado e a ativação dos sistemas enzimáticos produtores de corpos cetônicos. Uma vez que esse sistema esteja ativado, a taxa de produção de corpos cetônicos passa a ser conseqüência do fornecimento de FFA ao fígado. Do ponto de vista qualitativo, as adaptações metabólicas a um jejum, levando à cetose, não diferem da cetose da CAD. O que diferencia as duas situações é a maciça produção de FFA nesta última condição, conseqüência da falta de insulina, levando a concentrações muito elevadas de corpos cetônicos.

No interior do adipócito, é a lipase hormônio sensível (LHS) a responsável pela lipólise e esta lipase é inibida por insulina e adenosina, mas ativada pelos hormônios contra-reguladores.

A oxidação de ácidos graxos necessita do transporte para o interior da mitocôndria e a ativação da beta-oxidação, que cliva os FFA a acetil Co-A. O transporte é o fator limitante desse processo, já que, uma vez que o FFA entre na mitocôndria, ele é rapidamente convertido a acetil Co-A. Como as longas cadeias de ácidos graxos acetil Co-A não conseguem atravessar a membrana mitocondrial, deve ocorrer um passo de transesterificação, no qual a Co-A é trocada por carnitina. A enzima que faz essa transesterificação é a carnitina palmitoil transferase 1 (CPT 1). A atividade da CPT l é controlada pela malonil-CoA: em situações catabólicas (como na CAD), ocorre queda da malonil-CoA, o que libera a CPT 1 para transportar ácidos graxos para o interior da mitocôndria e a sua oxidação a corpos cetônicos. O glucagon medeia em parte a queda da malonil Co-A, tornando-se um propiciador de cetose (Figura 1) (6).

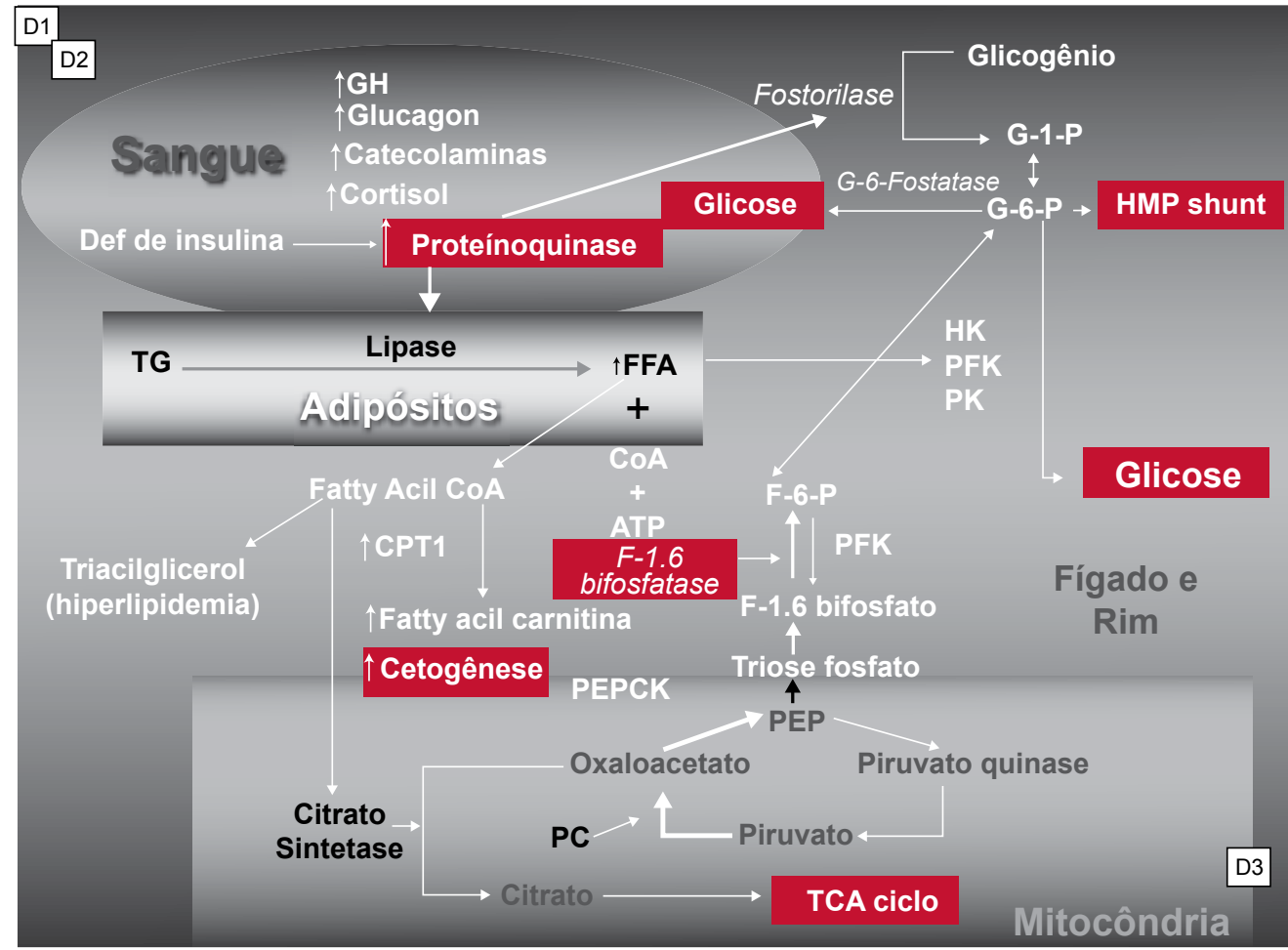

HK - hexoquinase; PFK - fosfofrutoquinase; PK - piruvato quinase; PEPCK - fosfoenolpiruvato carbóxi quinase; CPT 1 - carnitina palmitoil transferase 1; HMP shunt - via da hexosequinase; TCA ciclo - ciclo dos ácidos tricarboxilicos.

Figura 1. Mecanismos fisiopatológicos envolvidos na gênese da CAD. A falta de insulina desencadeia uma série de efeitos, aumentando a ação de proteínoquinases que ativam a LHS. Esta enzima converte triglicérides (TG) a FFA que, associandose a CoA formam as acil-coenzima A. Como a oxidação de glicose está comprometida, não há geração de malonil coenzima A e libera-se a ação da CPT 1, que transporta as acil-CoA para o interior da mitocôndria, onde ocorre a oxidação de ácidos graxos, gerando-se corpos cetônicos (6). 
A complicação clínica mais séria em uma CAD é o edema cerebral, de modo que não se está exagerando se se disser que o objetivo primordial do tratamento da CAD é evitar-se o edema cerebral. Pode-se dizer que a incidência de edema cerebral em episódios de CAD varia de $0,7 \%$ a $4,3 \%$ e a mortalidade varia de $30 \%$ a $64 \%$ (3). Em um estudo canadense, $19 \%$ dos casos de edema cerebral estavam presentes antes do início do tratamento (7). Estudos populacionais realizados nos Estados Unidos, no Canadá e na Inglaterra mostram mortalidade por edema cerebral variando de $21 \%$ a $24 \%$, e seqüelas neurológicas permanentes de $8 \%$ a $26 \%$ (7-9). Idade inferior a 5 anos, gravidade da acidose metabólica, grau de hipocapnia e elevação de uréia são fatores de risco de edema cerebral em CAD (1). Fica difícil avaliar se são fatores primários ou secundários a alguma alteração de base. Por exemplo, dizer que o grau de hipocapnia associa-se a edema cerebral é dizer que o grau de acidose metabólica pode ser o fator primário e a hipocapnia apenas a resposta do organismo a este distúrbio ácido básico. Da mesma forma, a infusão de bicarbonato de sódio está associada a maior incidência de edema cerebral (8), mas o processo básico pode ter sido a intensidade da acidose, que motivou o uso de bicarbonato.

São três os mecanismos envolvidos no edema cerebral: vasogênico, por causa da quebra da barreira hêmatoliquórica (como ocorre em traumas ou em tumores), citotóxico, em que ocorre envenenamento ou desarranjo metabólico, e osmótico, como ocorre na hiponatremia (Figura 2). Na CAD, o mecanismo do edema cerebral envolve vários dos componentes anteriormente mencionados e não deve ser o mesmo para todos os pacientes. $\mathrm{O}$ mecanismo predominante, avaliado por estudos de perfusão com ressonância nuclear magnética, é o vasogênico (10). É provável que a resposta do sistema nervoso central (SNC) inicial à CAD seja o edema vasogênico, enquanto o edema cerebral sintomático pode requerer hipóxia ou insulto posterior (liberação inadequada de vasopressina, sobrecarga fluida, hipoxia central por causa da rápida correção da acidose periféri-

\section{Patogênese do edema cerebral na CAD}

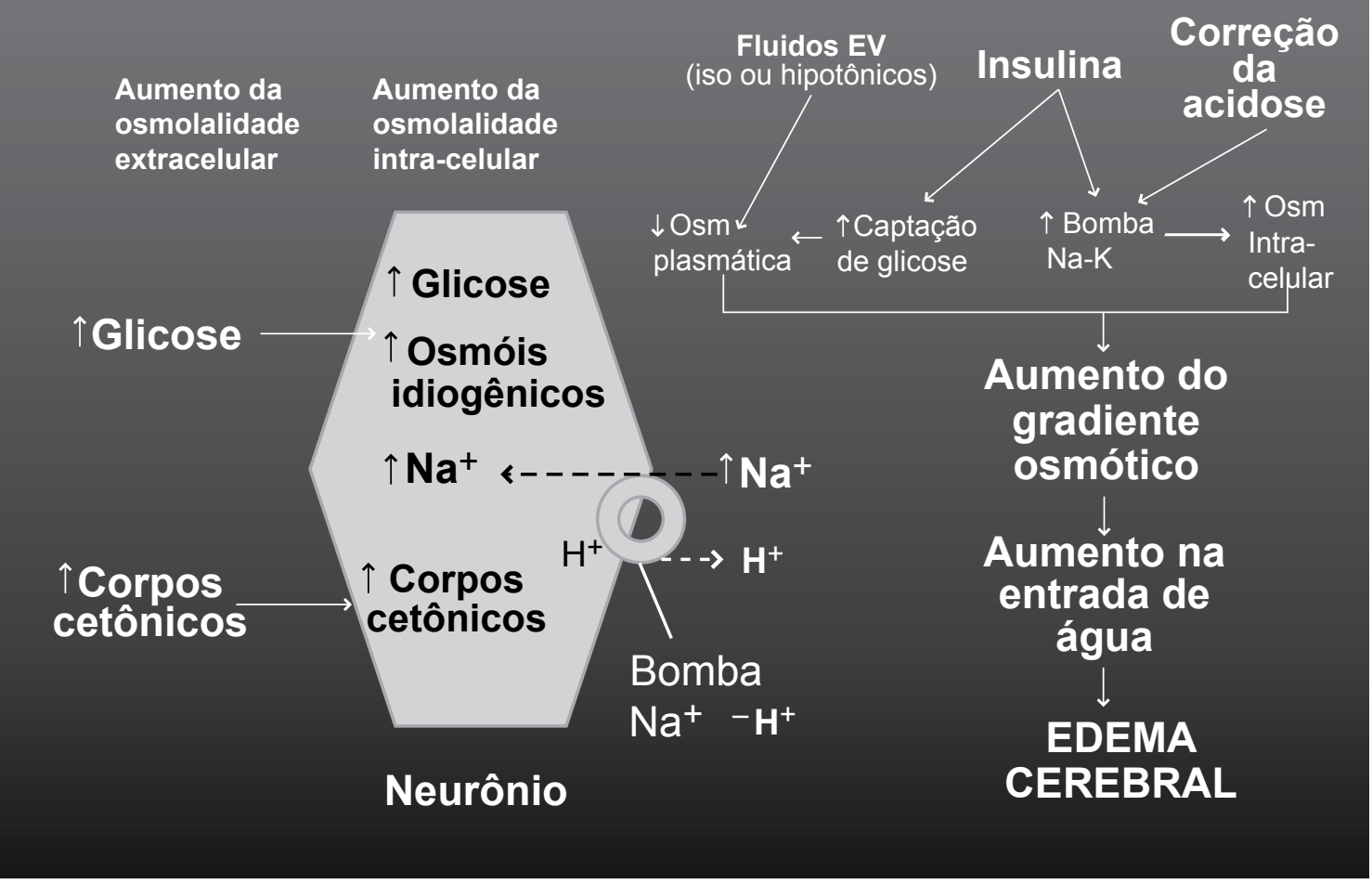

Figura 2. Representação esquemática de mecanismos que levam a edema cerebral na CAD. 
ca com administração de bicarbonato) que completa o quadro clínico (11). O desenvolvimento de edema cerebral apresenta distribuição bimodal, ocorrendo, dois terços dos casos nas primeiras seis a sete horas e o terço restante, 10 a 24 horas após o início do tratamento.

\section{QUADRO CLÍNICO}

O quadro clínico do DMl depende da idade em que a doença incide: a apresentação mais típica, com poliúria, polidípsia, polifagia e perda de peso é mais comum em crianças maiores. Em crianças de baixa idade (abaixo de 3 anos), muitas vezes os sintomas não são tão claros ou são difíceis de serem avaliados. Uma criança que ainda usa fralda pode ter poliúria, mas é difícil avaliá-la ou detectá-la. Em crianças no primeiro ano de vida, irritabilidade, desconforto, agitação, podendo progredir a torpor e coma podem ser detectados e o diagnóstico de DM pode não ficar evidente em um primeiro momento. Sempre que uma criança já tenha controle esfincteriano, mas volta a apresentar enurese, a suspeita de DM deve ser levantada. Muitas vezes, tal sintoma é atribuído a distúrbios emocionais e o quadro segue sem diagnóstico até a instalação de uma CAD. Infecções de pele (piodermites) repetidas, monilíase vaginal e/ou vulvar também devem chamar a atenção para o diagnóstico. Em um quadro de CAD, a acidose metabólica evidencia-se pela respiração acidótica (Kussmaul) e por um hálito cetônico, comparado ao odor de maçã verde. A desidratação é, em geral, equivalente à perda de $10 \%$ do peso e por ser hipertônica, é clinicamente menos evidente, ou seja, quando se calcula a reidratação, muitas vezes o cálculo é subestimado, estando a criança mais depletada em volume do que parecia clinicamente. Nunca se deve deixar de procurar um foco infeccioso como causa da descompensação e a alteração mental (obnubilação, torpor e coma) pode decorrer de um quadro meníngeo e não do desequilíbrio metabólico: sempre que o quadro mental não melhora com a volta gradual da criança ao seu estado metabólico normal, suspeite-se de meningite associada ou do desenvolvimento de edema cerebral. Esta possibilidade, uma vez aventada, deve ser imediatamente tratada (manitol), já que a evolução e eventuais seqüelas dependem da intervenção rápida no processo. Outras complicações neurológicas que não o edema cerebral também podem estar presentes e incluem hemorragia subaracnóide, trombose de artéria basilar, trombose venosa cerebral e coagulação intravascular disseminada. A Tabela 1 lista critérios diagnós-ticos para alterações neurológicas em crianças e adolescentes com CAD (3).
Tabela 1. Avaliação do estado neurológico em pacientes com CAD (Rosenbloom (3))

\section{Critérios diagnósticos}

Resposta motora ou verbal anormal à dor.

Postura decorticada ou decerebrada.

Paralisia de pares cranianos (especialmente III, IV, VI).

Padrão respiratório neurogênico anormal.

\section{Critérios maiores}

Capacidade mental alterada/nível de consciência oscilante.

Desaceleração mantida de freqüência cardíaca (redução de mais de 20 batimentos por minuto) não atribuivel a aumento de volume intravascular ou ao estado de sono.

Incontinência inapropriada para a idade.

\section{Critérios menores}

Vômitos após o tratamento inicial e sua cessação, se presente na internação.

Cefaléia (recorrente e mais intensa do que à admissão).

Letargia ou com dificuldade de ser despertado.

Pressão diastólica superior a $90 \mathrm{mmHg}$.

Idade inferior a 5 anos.

Rabdomiólise é um acompanhante freqüente dos HHS, mas algum grau de miólise (evidenciado por elevação de $\mathrm{CPK}$ ) ocorre com freqüência na CAD. Pancreatite aguda é uma complicação reconhecida em CAD de adultos, mas pode ser uma ocorrência freqüente e grave em crianças com HHS no início de um DM2. Em 50 crianças com CAD avaliadas no início de sua descompensação cetoacidótica, foi comum a observação de amilase e lipase elevadas. Dor abdominal que não se resolve com a correção da acidose deve levantar a suspeita de pancreatite aguda (12). Trombose venosa periférica já foi documentada em pacientes com CAD que requereram cateter femoral, o que sugere que a CAD seja um fator predisponente para diátese trombogênica, além do aspecto da desidratação que é, sabidamente, um fator contribuinte a esta entidade nosológica. Anormalidades de fatores de coagulação não têm sido demonstradas em pacientes com DMl na ausência de CAD, mas a atividade do fator von Willebrand permanece elevada 120 horas após a admissão do paciente em CAD (13).

DM antes dos 6 meses de idade é incomum. Em crianças pequenas para a idade gestacional pode desenvolver-se um DM transitório, em geral sem cetose, que 
tende a regredir em semanas ou meses, mas que necessita, na sua fase de descompensação glicêmica, do uso de insulina. Parece que nesses casos, há retardo na maturação das ilhotas pancreáticas e, usualmente, não se desenvolve DM permanente. Raramente, a agenesia pancreática pode ser responsável pelo DM bastante precoce. Síndromes de resistência à insulina (leprechaunismo, por exemplo, caracterizadas por retardo de crescimento intra-uterino, hipoglicemia de jejum e hiperglicemia pós-prandial, associadas à enorme resistência à insulina) podem se manifestar precocemente como DM.

\section{DIAGNÓSTICOS DIFERENCIAIS}

Algumas situações clínicas podem levar a quadro clínico semelhante ao de uma CAD. Quando se atende um paciente com história prévia de DM a suspeita da descompensação em cetoacidose torna-se mais clara, mas, em um paciente até então hígido, alguns diferenciais devem ser salientados. Em termos do desarranjo metabólico, as hipoglicemias, a encefalopatia hepática ou urêmica, os erros inatos do metabolismo, o desequilíbrio hidroeletrolítico (doença de Addison, diabetes insípido, intoxicação hídrica), a acidose lática (intoxicação por salicilato), as drogas ilícitas, a hipóxia ( $\mathrm{CO}$, cianeto) ou os quadros pós-ictais podem confundir o diagnóstico. Quando ocorre alteração de sensório e mesmo coma, lembrar de meningite, encefalite, trauma/concussão, hemorragia (extradural, subdural, subaracnóide ou intracerebral), tumor cerebral, abscesso cerebral ou trombose cerebral. Exames de imagem e bioquímicos ajudam a elucidar o processo e conduzir a condutas adequadas a cada caso.

\section{TRATAMENTO DA CAD}

Classicamente, o tratamento da CAD tem sido feito com infusão endovenosa de insulina regular, ao lado de medidas de reposição de volume, detecção do fator desencadeante (infecção, falta de administração de insulina, excesso alimentar). Com o objetivo de simplificar o tratamento, compara-se a evolução de pacientes em CAD tratados da maneira clássica, com uma proposta em que insulina ultra-rápida (lispro) era administrada por via subcutânea a cada duas horas. Estudaram-se 60 episódios de CAD admitidos em nosso pronto-socorro e, aleatoriamente, os pacientes foram submetidos à infusão contínua de insulina regu- lar (grupo IRIC $-\mathrm{n}=30$ ) ou insulina lispro subcutânea a cada duas horas (grupo lispro $-\mathrm{n}=30$ ). Pacientes que necessitavam de procedimentos cirúrgicos ou em uso de glicocorticóides ou agentes imunossupressores foram excluídos do estudo (5).

As características laboratoriais à admissão foram semelhantes em ambos os grupos, com glicemia média de $24,6 \pm 7,9 \mathrm{mmol} / \mathrm{L}(442,8 \pm 142,2)$ no grupo lispro e 24,6 $\pm 8,1 \mathrm{mmol} / \mathrm{L}$ no grupo IRIC, osmolalidade de $311,95 \pm 13,71$ no grupo 1 ispro e $312,91 \pm 13,81$ no grupo IRIC (Tabela 2 ).

$\mathrm{Na}$ sala de emergência, todos os pacientes tiveram um cateter venoso para infusão de fluidos (solução fisiológica $-\mathrm{NaCl}$ a $0,9 \%$ ) e coleta de amostras de sangue para exames. A infusão inicial foi de $20 \mathrm{~mL} / \mathrm{kg}$ na primeira hora, para restabelecer a perfusão periférica. Em alguns casos, esta dose foi repetida, até se ter uma melhora da perfusão. A partir daí, soro fisiológico foi infundido na velocidade de $10 \mathrm{~mL} / \mathrm{kg} /$ hora até a reparação do volume. Um paciente em CAD tem perda de volume inicial estimada em $10 \%$ do peso, de modo que, em princípio, programou-se essa reparação em 10 horas, sempre com reavaliações freqüentes. Após micção, potássio foi adicionado à solução de reparação para a concentração de $20 \mathrm{mEq} / \mathrm{L}$, para manter calemia superior a $3,8 \mathrm{mEq} / \mathrm{L}$. Adiava-se a reposição de potássio se a calemia fosse superior a $6,5 \mathrm{mEq} / \mathrm{L}$. Ressaltar que sempre há depleção de potássio na CAD mas, por causa da acidose, ocorre troca de $\mathrm{H}^{+}$por $\mathrm{K}^{+} \mathrm{e}$ os níveis séricos podem estar falsamente elevados. Procurou-se evitar a administração de bicarbonato, que tem sido correlacionado ao desenvolvimento de edema cerebral. Somente quando $\mathrm{pH}<7,0$ ou bicarbonato sérico inferior a $5 \mathrm{mmol} / \mathrm{L}$, uma pequena dose ( $1 \mathrm{mmol} / \mathrm{kg} /$ hora) de bicarbonato de sódio foi administrada. Fosfato foi administrado se a fosfatemia estivesse $<0,5 \mathrm{mmol} / \mathrm{L}$. Se o paciente ainda não tinha condições de se alimentar, quando a glicemia atingia $250 \mathrm{mg} / \mathrm{dL}$, adicionava-se soro glicosado a $5 \%$ ao regime de fluidos.

\section{Insulinoterapia}

Administração de insulina foi iniciada 1 a 2 horas após o início da hidratação, na dose de $0,15 \mathrm{U} / \mathrm{kg}$ a cada 2 horas no grupo lispro e $0,1 \mathrm{U} / \mathrm{kg} /$ hora no grupo IRIC. Glicemia capilar era coletada a cada hora e, com este ritmo de infusão, prevê-se queda de 60 a $90 \mathrm{mg} /$ $\mathrm{dL} /$ hora na glicemia. Quedas inferiores a $60 \mathrm{mg} / \mathrm{dL} / \mathrm{h}$ 
Tabela 2. Características dos pacientes e valores bioquímicos iniciais em 60 episódios de CAD ocorridos em 46 pacientes.

\begin{tabular}{lcc}
\hline & Grupo lispro & Grupo IRIC \\
\hline Idade (anos) & $11,3 \pm 3,6$ & $12,1 \pm 3,3$ \\
Peso (kg) & $39,06 \pm 12,86$ & $41,6 \pm 12,2$ \\
\hline Masculino/feminino & $8 / 17$ & $5 / 16$ \\
$\mathrm{pH}$ & $7,17 \pm 0,10$ & $7,18 \pm 0,10$ \\
Ânion Gap (mmol/L) & $22,35 \pm 7,23$ & $29,55 \pm 9,04$ \\
Beta-hidróxibutirato (mmol/L) & $8,45 \pm 2,5$ \\
Glicemia (mg/dL) & $8,16 \pm 3,53$ & $442,8 \pm 145,8$ \\
\hline Na (mmol/L) & $442,2 \pm 142,2$ & $136,68 \pm 4,09$ \\
K (mmol/L) & $137,09 \pm 4,79$ & $5,08 \pm 0,77$ \\
Cl (mmol/L) & $5,11 \pm 0,61$ & $105,13 \pm 9,37$ \\
Bicarbonato (mmol/L) & $109,86 \pm 6,56$ & $9,08 \pm 3,61$ \\
\hline Osmolalidade (mosm/kg) & $10,38 \pm 4,47$ & $312,91 \pm 13,81$ \\
\hline
\end{tabular}

exigem que se dobre a dose de insulina e quedas superiores a $100 \mathrm{mg} / \mathrm{dL} / \mathrm{h}$ recomendam reduzir-se à metade as doses de insulina. Importante vigiar se a evolução do nível de consciência que, com um tratamento eficaz, melhora progressivamente. Salientar que, em caso de piora neurológica, uma das possibilidades é o edema cerebral que deve ser imediatamente tratado com infusão de manitol (manitol a $20 \%-1 \mathrm{~g} / \mathrm{kg}$ endoveno- so). Os resultados comparativos entre os dois grupos (Tabela 3) mostraram evoluções semelhantes em todos os parâmetros avaliados, o que autoriza a recomendar o uso de insulina de ação ultra-rápida, via subcutânea, para o tratamento de CAD, com uma importante simplificação técnica e redução de custos do tratamento, evitando-se a necessidade de uma segunda veia (uma para infusão de insulina e outra para administração de

Tabela 3. Características bioquímicas dos grupos lispro e IRIC durante o tratamento da CAD.

\begin{tabular}{|c|c|c|c|c|c|c|}
\hline & $\begin{array}{l}\text { Glicemia } \\
\text { (mg/dL) }\end{array}$ & $\mathrm{pH}$ & $\begin{array}{l}\text { Bicarbonato } \\
(\mathrm{mmol} / \mathrm{L})\end{array}$ & $\begin{array}{l}\beta \text {-hidróxibutirato } \\
\text { (mmol/L) }\end{array}$ & Ânion Gap & $\begin{array}{c}\text { Osm } \\
\text { (mOsm/kg) }\end{array}$ \\
\hline $\begin{array}{l}\text { Inicial } \\
\text { IRIC }\end{array}$ & $442,8 \pm 145,8$ & $7,18 \pm 0,1$ & $9,0 \pm 3,6$ & $8,4 \pm 2,7$ & 295 & $312 \pm 13$ \\
\hline Lispro & $442,8 \pm 142,2$ & $7,17 \pm 0,1$ & $10,3 \pm 4,7$ & $8,1 \pm 3,5$ & 22,3 & $311 \pm 13$ \\
\hline $\begin{array}{l}\text { Após } 2 \text { horas } \\
\text { IRIC }\end{array}$ & $333,0 \pm 73,8$ & $7,1 \pm 0,1$ & $9,7 \pm 4,0$ & $7,0 \pm 2,5$ & 25,4 & $310 \pm 12$ \\
\hline Lispro & $334,8 \pm 126,0$ & $7,1 \pm 0,1$ & $9,8 \pm 5,7$ & $7,5 \pm 3,5$ & 25,1 & $305 \pm 16$ \\
\hline $\begin{array}{l}G=250 \\
\text { IRIC }\end{array}$ & $223,2 \pm 41,4$ & $7,2 \pm 0,1$ & $9,7 \pm 4,0$ & $4,0 \pm 2,5$ & 19,3 & $305 \pm 13$ \\
\hline LIspro & $205,2 \pm 66,6$ & $7,2 \pm 0,1$ & $9,8 \pm 5,7$ & $5,1 \pm 3,1$ & 17,6 & $302 \pm 13$ \\
\hline $\begin{array}{l}6 \text { horas após } \\
\text { IRIC }\end{array}$ & $187,2 \pm 86,4$ & $7,3 \pm 0,1$ & $15,7 \pm 4,4$ & $2,6 \pm 2,1$ & 14,5 & $297 \pm 11$ \\
\hline Lispro & $241,2 \pm 82,8$ & $7,3 \pm 0,1$ & $14,2 \pm 6,1$ & $3,2 \pm 2,8$ & 17,2 & $296 \pm 10$ \\
\hline $\begin{array}{l}24 \text { horas após } \\
\text { IRIC }\end{array}$ & $270,0 \pm 133,2$ & $7,3 \pm 0,07$ & $19,5 \pm 5,1$ & $0,8 \pm 0,9$ & 13,5 & $302 \pm 10$ \\
\hline Lispro & $246,6 \pm 91,8$ & $7,3 \pm 0,06$ & $18,1 \pm 4,7$ & $1,1 \pm 1,5$ & 14,5 & $297 \pm 5$ \\
\hline
\end{tabular}

$\mathrm{G}=250$ - momento em que a glicemia atingiu $250 \mathrm{mg} / \mathrm{dL} ; 6$ horas após - 6 horas após a glicemia ter atingido $250 \mathrm{mg} / \mathrm{dL}$

24 horas após -24 horas após a glicemia ter atingido $250 \mathrm{mg} / \mathrm{dL}$. 
fluidos) ou de bomba de infusão de insulina. O tempo necessário para os pacientes atingirem glicemia de $250 \mathrm{mg} / \mathrm{dL}$ foi de seis horas em ambos os grupos. Acidose metabólica e cetose resolveram-se adequadamente em ambos os grupos.

Um dado interessante é que a osmolalidade sérica permaneceu elevada, em ambos os grupos, mesmo após 24 horas do início do tratamento. Antes de se achar que isso é uma falha terapêutica, pode-se especular que tal achado pode propiciar proteção contra o edema cerebral, já que não ocorrem mudanças rápidas de osmolalidade e, portanto, não ocorrem desvios rápidos de volume de um compartimento a outro. Não houve nenhum caso de edema cerebral em nossos pacientes.

\section{CONSIDERAÇÕES FINAIS}

Os estados hiperglicêmicos no DMl assumem gravidade quando resultam $\mathrm{CAD}$ ou $\mathrm{HHS}$, este último, mais freqüentemente associado ao DM2. O tratamento de uma CAD exige conhecimento fisiopatológico da condição e deve ser realizado em ambiente hospitalar. Ainda hoje, a mortalidade decorrente de CAD é da ordem de 5\% e, quando se instala o edema cerebral $(0,3 \%$ a $4,3 \%$ dos casos) a mortalidade sobe a $30 \%$ ou mais. O tratamento da CAD e do HHS é semelhante, ressaltando que nas condições hiperglicêmicas hiperosmolares não há acidose ou cetose importantes e as correções glicêmicas devem ser lentas para evitar grandes e rápidos deslocamentos fluídicos entre os vários compartimentos do organismo, com possibilidade de dano cerebral. A utilização de insulina de ação ultra-rápida por via subcutânea trouxe importante simplificação técnica ao tratamento e permite prever uma queda glicêmica de 60 a $90 \mathrm{mg} / \mathrm{dL} /$ hora com a dose de $0,15 \mathrm{U} / \mathrm{kg} / \mathrm{a}$ cada 2 horas. Importante salientar que o médico deve estar ao lado do paciente o tempo todo, já que modificações nas infusões de insulina e de fluidos muitas vezes não são previsíveis e devem ser tomadas no momento em que ocorrem. Não é prudente deixar o paciente com CAD ou HHS com uma prescrição preestabelecida e transferir o controle do paciente à equipe paramédica. A detecção precoce de desvios de comportamento metabólico é fundamental para que se evitem complicações. A mais temida dessas complicações é o edema cerebral. Uma vez suspeitado, não há indicação de exames de imagem ou de mais dosagens laboratoriais para confirmação diagnóstica, mas é fundamental que medidas efetivas sejam imediatamente tomadas, como infusão de manitol e vigilância do estado de consciência do paciente. As seqüelas neurológicas e os eventuais óbitos ocorrem com freqüência alta e todo o esforço deve ser empregado para se tentar evitar tal desfecho. Salientar sempre que tratar complicações hiperglicêmicas no DMl, especialmente $\mathrm{CAD}$, exigem que se tomem medidas com rapidez e segurança, mas sem pressa!

\section{REFERÊNCIAS}

1. Dunger DB, Sperling MA, Acerini $C L$, Bohn DJ, Daneman $D$, Danne TPA, et al. European Society for Paediatric Endocrinology/Lawson Wilkins Pediatric Endocrine Society consensus statement on diabetic ketoacidosis in children and adolescents. Pediatrics. 2004;113:e133-140.

2. Fourtner SH, Weinzimer SA, Katz LEL. Hyperglycemic hyperosmolar nonketotic syndrome in children with type 2 diabetes. Pediatr Diabetes. 2005;6:129-35.

3. Rosenbloom AL. Hyperglycemic crises and their complications in children. J Ped Endocrinol Metab. 2007;20:5-18.

4. Damiani D, Damiani D. Diabetes mellitus na infância: diagnóstico e tratamento. Pediatria Moderna. 2007;43(5):205-24.

5. Della Manna T, Steinmetz L, Campos P, Farhat S, Schvartsman $\mathrm{C}$, Kuperman $\mathrm{H}$, et al. Subcutaneous use of a fast-acting insulin analog: an alternative treatment for pediatric patients with diabetic ketoacidosis. Diabetes Care. 2005;28(8):1856-61.

6. Kltabchi AE, Nyenewe EA. Hyperglycemic crisis in diabetes mellitus: diabetic ketoacidosis and hyperglycemic hyperosmolar state. Endocrinol Metab Clin N Am. 2006;3:725-51.

7. Lawrence SE, Cummings EA, Gaboury I, Daneman D. Poçulationbased study of incidence and risk factors for cerebral edema in pediatric diabetic ketoacidosis. J Pediatr. 2005;146:688-92.

8. Glaser N, Barnett P, McCaslin I, Nelson D, Trainor J, Louie J, et al. Risk factors for cerebral edema in children with diabetic ketoacidosis. N Engl J Med. 2001;344:264-9.

9. Edge JA, Ford-Adams ME, Dunger DB. Causes of death in children with insulin dependent diabetes 1990-96. Arch Dis Child. 1999;81:318-23.

10. Glaser N, Wooten-Gorges SL, Buonocore MH, Marcin JP, Rewers A, Strain J, et al. Frequency of subclinical cerebral edema in children with diabetic ketoacidosis. Pediatr Diabetes. 2006;7:75-80.

11. Levitsky LL. Symptomatic cerebral edema in diabetic ketoacidosis: the mechanism is clarified but still far from clear [Editorial]. J Pediatr. 2004;145:149-150.

12. Haddad NG, Croffie JM, Rugster EA. Pancreatic enzyme elevations in children with diabetic ketoacidosis. J Pediatr. 2004;145:122-4.

13. Carl CF, Hoffman WH, Passmore GG, Truemper EJ, Lightsey $\mathrm{AL}$, Cornwell PE, et al. Diabetic ketoacidosis promotes a prothrombotic state. Endocrinol Res 2003;29:73-82.

Endereço para correspondência:

Durval Damiani

Rua Bela Cintra, 2117, apto. 9

01415-002 Cerqueira César, São Paulo, SP

E-mail: durvald@iconet.com.br 